\title{
CAPACIDADE ANTIOXIDANTE E QUALIDADE PÓS-COLHEITA DE ABACATE 'FUERTE'
}

\author{
ROGÉRIO LOPES VIEITES ${ }^{2}$, ÉRICA REGINA DAIUTO³ ${ }^{3}$ JOANA GIFFONI FIGUEIREDO FUMES ${ }^{4}$
}

RESUMO-A capacidade antioxidante e a qualidade pós-colheita do abacate 'Fuerte' foram avaliadas. Os frutos foram mantidos sob temperatura ambiente $\left(24 \pm 1{ }^{\circ} \mathrm{C}\right)$ e sob refrigeração em $10 \pm 1{ }^{\circ} \mathrm{C}$ e $90 \pm 5 \%$ UR e avaliados durante 15 dias. Determinaram-se a perda de massa e a taxa respiratória. As características físicoquímicas avaliadas foram acidez total titulável (AT), sólidos solúveis (SS), ratio, $\mathrm{pH}$, firmeza, atividade da enzima polifenoloxidase (PPO), cor ( $\left.\mathrm{L}, \mathrm{a}^{*} \mathrm{e} \mathrm{b}^{*}\right)$, fenólicos totais e atividade antioxidante (AA) por DPPH . Nas duas condições de armazenamento, o pico respiratório ocorreu no $9^{\circ}$ dia.Os frutos mantidos sob temperatura refrigerada apresentaram menor perda de massa e maior firmeza em relação àqueles em temperatura ambiente. O comportamento respiratório refletiu na redução dos valores de AT e no conteúdo de SS que diminuíram a partir do pico de respiração. Os valores de $\mathrm{pH}$ aumentaram como consequência da diminuição da AT. Os valores de luminosidade mantiveram-se elevados, observando-se diminuição do componente de cor verde na polpa com o armazenamento. A atividade da PPO diminuiu com o armazenamento. Os valores de AA variaram de 17,6 a 68,7\%. O conteúdo de fenólicos totais diminuiu após o pico respiratório, e valores crescentes de AA foram observados mesmo após o $9^{\circ}$ dia.

Termos para indexação: Persea americana Mill., atividade anti radical livre, refrigeração, compostos fenólicos.

\section{ANTIOXIDANT CAPACITY AND POSTHARVEST QUALITY OF 'FUERTE' AVOCADO}

ABSTRACT - Antioxidant capacity and quality of the avocado 'Fuerte' were evaluated. The fruits were maintained under room temperature $\left(24 \pm 1^{\circ} \mathrm{C}\right)$ and refrigeration $\left(10 \pm 1^{\circ} \mathrm{C}\right.$ and $90 \pm 5 \%$ UR $)$ during 15 days. Weight loss and respiratory rate were evaluated. Physical and chemical characteristics were determined: titratable acidity (TA), soluble solids (SS), ratio, $\mathrm{pH}$, firmness, polyphenoloxidase (PPO) activity, color (L, a* and $\mathrm{b}^{*}$ ), total phenolics and antioxidant activity (AA) for DPPH $\cdot$. In the two storage conditions the breathing pick happened in the 9th day. The fruits maintained under refrigerated temperature presented smaller mass loss and larger firmness than those in room temperature. The respiratory behavior result in the values of TA and content of SS that decreased starting from respiratory pick. The $\mathrm{pH}$ values increased as a consequence of the decrease of the TA. Brightness values stayed high, being observed a decrease of the green component color and increase of the yellow in the pulp with the storage PPO activity decreased with the storage. The values of AA varied from 17,6 to $68,7 \%$. Total phenolics content decreases after the breathing pick and growing values of AA were observed even after the 9th day.

Index terms: Persea americana Mill., free radical scavenger activity, refrigeration, total phenolics.

\section{INTRODUÇÃO}

O abacate (Persea americana Mill.) possui considerável qualidade nutritiva, com alto conteúdo de fibras, proteínas, sais minerais, destacando-se o potássio e vitaminas, especialmente a vitamina $\mathrm{E}$ (USDA, 2007), possui também significativa quantidade de ácidos graxos insaturados com efeitos benéficos na prevenção de doenças cardiovasculares (TANGO et al., 2004). Estudos têm demonstrado que o fruto possui compostos lipofílicos anticancerígenos como carotenoides (DING et al., 2007).

Wang, Terrell e Bostic (2010) mencionam que estudos sobre a composição fitoquímica de abacates são escassos e não há conhecimento sobre o teor de compostos fenólicos totais e capacidade antioxidante em diferentes raças e cultivares de abacate. Vale ressaltar que estes autores realizaram o estudo para determinação de capacidade antioxidante na polpa, semente e casca de diversas variedades de abacate,

${ }^{1}$ (Trabalho 068-11). Recebido em: 08-02-12. Aceito para publicação em: 29-09-2012. Projeto Financiado pela CAPES e FAPESP. ${ }^{2}$ Docente Departamentos de Gestão e Tecnologia Agroindustrial da Faculdade de Ciências Agronômicas da UNESP-Botucatu. E -mail: vieites@fca.unesp.br 
exceto a 'Fuerte'. Abacates 'Fuerte' são frutos com calibre menor e valorizados no mercado externo, principalmente Europa e EUA.

Os antioxidantes são compostos que atuam inibindo e/ou diminuindo os efeitos desencadeados pelos radicais livres (SOARES et al., 2005), podendo ser definidos como compostos que protegem as células contra os efeitos danosos dos radicais livres oxigenados e nitrogenados, formados nos processos oxidativos. Os radicais livres em excesso geram um desbalanço, dando início ao estresse oxidativo, processo metabólico responsável pelo desencadeamento de diversos tipos de doenças crônico-degenerativas. Os antioxidantes podem ser obtidos por meio da ingestão de alimentos, destacando- se as vitaminas E e C, os carotenoides, os compostos fenólicos, entre outros (ALI et al., 2008).

Os compostos fenólicos são os maiores responsáveis pela atividade antioxidante em frutos, fazendo destes uma fonte natural de antioxidantes (HEIM et al., 2002). Entretanto, o conteúdo de compostos fenólicos em alimentos vegetais depende de um número de fatores intrínsecos, como gênero, espécie e cultivar, e extrínsecos, como agronômico, ambiental, manuseio e armazenamento (TOMÁSBARBERÁN; ESPÍN, 2001).

O abacate é fruto climatérico cujo amadurecimento ocorre poucos dias após a colheita (HARDENBURG et al., 1986; SEYMOUR; TUCKER, 1993), e o comportamento pós-colheita pode ser influenciado pela temperatura e pelo tempo de armazenamento (TEIXEIRA et al., 1991). A temperatura adequada e o período para a conservação do abacate variam com a raça e a cultivar (ZAUBERMAN et al., 1973), pois estas apresentam sensibilidade diferente aos danos pelo frio. Honório e Moretti (2002) relatam que a temperatura mínima para armazenamento desta fruta varia de $5^{\circ} \mathrm{C}$ a $12^{\circ} \mathrm{C}$, enquanto Chitarra e Chitarra (2005) citam que, dependendo da cultivar, esta temperatura varia de $4,5^{\circ} \mathrm{C}$ a $13^{\circ} \mathrm{C}$. A literatura aponta diversos estudos relacionados ao aumento do período de conservação de abacate, como avaliação da temperatura de armazenamento, uso de atmosfera modificada com aplicação de cera, irradiação gama e tratamento térmico para prevenção de sintomas de injúria pelo frio (CASTRO; BLEIROTH,1982; SEYMOUR; TUCKER, 1993; GERMANO; ARTHUR;WIENDL, 1996; OLIVEIRA et al., 2000; DONADON, 2009).

Atualmente, a avaliação da capacidade antioxidante tem sido realizada para determinar a eficiência dos antioxidantes naturais em relação à proteção do produto vegetal contra oxidação e perda do valor comercial e nutricional. Os parâmetros de qualidade pós-colheita comumente realizados são perda de massa, firmeza, ratio, perda de vitaminas, $\mathrm{pH}$, acidez total titulável, coloração, atividades de enzima de escurecimento, entre outros. A capacidade antioxidante em abacate 'Fuerte' e sua alteração com o armazenamento ainda não foram relatadas na literatura. Portanto, o objetivo desta pesquisa foi avaliar a capacidade antioxidante e a qualidade pós-colheita do abacate 'Fuerte'.

\section{MATERIAL E MÉTODOS}

Foram utilizados frutos de abacate 'Fuerte', da safra de 2009, fornecidos pela empresa Jaguacy, localizada em Bauru-SP, cujas coordenadas geográficas são: latitude $22^{\circ} 19^{\prime} 18^{\prime \prime} \mathrm{S}$, longitude $49^{\circ} 04^{\prime} 13$ " W e $526 \mathrm{~m}$ de altitude, distante $90 \mathrm{~km}$ de Botucatu: latitude de $22^{\circ} 52^{\prime} 20^{\prime \prime} \mathrm{S}$, longitude $48^{\circ} 26^{\prime} 37^{\prime \prime} \mathrm{W}$ e $815 \mathrm{~m}$ de altitude. Os frutos cuidadosamente colhidos no ponto de maturação fisiológica e de acordo com o teor de óleo foram selecionados para tornar o lote ainda mais homogêneo quanto ao tamanho, cor e ausência de injúrias e defeitos. Os frutos foram mantidos sob temperatura ambiente $\left(24 \pm 1^{\circ} \mathrm{C}\right)$ e sob refrigeração em câmara fria a $10 \pm 1{ }^{\circ} \mathrm{C}$ e $90 \pm 5 \%$ UR e avaliados durante 15 dias no intervalo de 3 dias. Em cada um destes tratamentos, foram avaliados 3 frutos por repetição, com 3 repetições

Foram realizadas as seguintes análises:

Perda de massa fresca, pela pesagem dos frutos em balança analítica, considerando a massa inicial de cada amostra, com os resultados expressos em percentagem.

A respiração foi determinada pela liberação de $\mathrm{CO}_{2}$ em cada embalagem, de acordo com metodologia adaptada de Bleinroth et al. (1976), utilizandose para isso de solução de hidróxido de bário saturado e solução de hidróxido de potássio $0,1 \mathrm{~N}$. Para tanto, foi utilizada a seguinte fórmula:

$$
\mathrm{TCO}_{2}=\frac{2,2\left(\mathrm{Vo}_{\mathrm{V}} \mathrm{V}_{1}\right) \cdot 10}{\text { P.T }}
$$

em que, $\left.\mathrm{Kg}^{-1} \cdot \mathrm{h}^{-1}\right)$

$\mathrm{TCO}_{2}=$ taxa de respiração $\left(\mathrm{mL}\right.$ de $\mathrm{CO}_{2}$.

$\mathrm{Vo}=$ volume gasto de $\mathrm{HCl}$ para titulação de hidróxido de potássio - padrão antes da absorção de $\mathrm{CO}_{2}(\mathrm{~mL})$;

$\mathrm{V}_{1}=$ volume gasto de $\mathrm{HCl}$ para titulação de hidróxido de potássio após a absorção de $\mathrm{CO}_{2}$ da respiração $(\mathrm{mL})$;

$\mathrm{P}=$ massa dos frutos;

$\mathrm{T}=$ tempo da respiração; 
2,2 = inerente ao equivalente de $\mathrm{CO}_{2}(44 / 2)$, multiplicado pela concentração do ácido clorídrico, e

$10=$ ajuste para o total de hidróxido de potássio utilizado.

Os teores de sólidos solúveis (SS), $\mathrm{pH}$ e acidez total titulável (AT) foram determinados seguindo as Normas Analíticas do Instituto Adolf Lutz (ZENEBON et al., 2008). O teor de sólidos solúveis foi medido, em leitura refratométrica em ${ }^{\circ}$ Brix, a $20^{\circ} \mathrm{C}$, com refratômetro digital, conforme metodologia. Foi determinado o ratio pela relação entre o teor de sólidos solúveis e a acidez titulável (TRESSLER; JOSLYN, 1961).

A avaliação da firmeza foi feita utilizando-se de texturômetro, com a distância de penetração de 20 $\mathrm{mm}$, velocidade de $2,0 \mathrm{~mm} \mathrm{seg}^{-1}$. e ponta de prova TA $9 / 1000$, os resultados foram apresentados em gramas força $^{-1}$. Foram realizadas 5 leituras para cada uma das 3 repetições em todos os tratamentos.

A atividade da enzima polifenoloxidase (PPO) foi determinada pelo método de Cano et al. (1997), e os resultados, expressos em UAE ${ }^{-1} \mathrm{~min}^{-1}$.

A coloração foi medida em colorímetro da marca Konica Minolta (Chroma meter, CR 400/410). A cor foi expressa pelo sistema de coordenadas retangulares $\mathrm{L} \mathrm{a}^{*} \mathrm{~b}^{*}$, conforme a CIE (Comission Internatinale de E'clairage), em que L expressa em porcentagem valores de luminosidade $(0 \%=$ negro e $100 \%=$ branco); $\mathrm{a}^{*}$ representa a intensidade de cor vermelha $(+)$ ou verde $(-)$, e b* a intensidade de cor amarela $(+)$ ou azul (-).

O conteúdo de compostos fenólicos totais foi determinado pelo método espectrofotométrico de Folin-Ciocalteau descrito por Singleton et al. (1999), utilizando ácido gálico como padrão, e os resultados, expressos em $\mu \mathrm{g}$ GAE. $100 \mathrm{~g}^{-1}$.

A capacidade antioxidante das amostras foi avaliada pelo DPPH (2,2-diphenyl-1-picrylhydrazyl), segundo Mensor et al., 2001. A atividade antirradical foi determinada na forma de Atividade Antioxidante (AA), pela equação:

$$
\begin{aligned}
& \mathrm{AA}(\%)=100-[(\mathrm{Aa}-\mathrm{Ab}) \times 100] / \mathrm{Ac}, \\
& \text { em que, }
\end{aligned}
$$

$\mathrm{Aa}=$ absorbância da amostra;

$\mathrm{Ab}=$ absorbância do branco;

$\mathrm{Ac}=$ absorbância do controle negativo.

$\mathrm{O}$ controle negativo foi feito substituindo-se o volume do extrato por igual volume do solvente utilizado na extração (etanol). O branco foi preparado substituindo o volume da solução de DPPH por igual volume de solvente

Os dados foram então submetidos à análise de regressão linear ou polinomial de segundo grau, conforme o caso. Utilizou-se a análise de variância, pelo teste de Tukey, para comparações múltiplas das médias, a um nível de significação de 5\%. Foi realizada também análise de correlação de Pearson para todos os parâmetros avaliados.

\section{RESULTADOS E DISCUSSÃO}

\section{Perda de massa fresca}

Os resultados para análise de perda de massa fresca dos frutos, representada por duas equações lineares (Figura 1), foram crescentes para as duas condições de armazenamento. No armazenamento refrigerado, a perda de massa não superou $2 \%$ do peso inicial dos frutos, entretanto a perda de massa foi mais acentuada nos frutos mantidos sob temperatura ambiente. Daiuto et al. (2010 b) observaram que nos frutos 'Hass' armazenados sob refrigeração, para qualquer tipo de tratamento físico realizado (radiação ou tratamento térmico), a perda de massa foi inferior nos frutos armazenados sob temperatura ambiente.

Vale ressaltar que, para a maioria dos produtos hortícolas frescos, a máxima perda de massa fresca tolerada para o não aparecimento de murcha e/ou enrugamento da superfície oscila entre 5 e $10 \%$ (FINGER; VIEIRA, 2002), e produtos perecíveis como o abacate, mesmo quando colocados em condições ideais, sofrem alguma perda de peso durante o armazenamento devido ao efeito combinado da respiração e da transpiração (CHITARRA; CHITARRA, 2005).

\section{Taxa respiratória}

Observou-se para taxa respiratória, nas duas condições de armazenamento, o comportamento climatérico descrito para o abacate (SEYMOUR; TUCKER, 1993; DAIUTO et al., 2010a; DAIUTO et al., 2010b). Os frutos armazenados sob temperatura ambiente apresentaram maior produção de $\mathrm{CO}_{2}$, quando em comparação aos mantidos sob refrigeração (Figura 2). Baseando-se no fato de que todo e qualquer processo respiratório é sempre de natureza degradativa, tendo como função primordial a produção de energia e intermediários metabólitos, pressupõe-se para esses frutos menor potencial de conservabilidade.

Pôde-se observar um aumento da taxa respiratória até o $9^{\circ}$ dia de armazenamento, concordando com Daiuto et al. (2010b) em abacate 'Hass', que constataram ainda um pico mais pronunciado no tratamento com radiação gama. Um aumento da taxa respiratória após este momento pode ser indicativo de que, em consequência da senescência dos frutos, houve ataque de microrganismo, sendo a respiração detectada em função deste fato. 
Manolopoulou e Papadopoulou (1998) afirmam que a intensidade da taxa respiratória está relacionada com a capacidade de armazenamento do produto, e que, quanto maior a taxa respiratória, menor é o tempo de armazenamento.

A temperatura é o maior fator no controle da respiração e da transpiração, bem como na deterioração microbiana, podendo interferir na velocidade de reação dos processos metabólicos, no tempo de armazenamento, e causar distúrbios fisiológicos nos frutos. A relação respiração/temperatura é governada pela lei de Vant Hoff, que considera um aumento de duas a três vezes na velocidade da atividade respiratória para cada aumento de $10^{\circ} \mathrm{C}$ na temperatura (KLUGE et al., 2002).

O tipo de metabolismo respiratório apresentado refletiu-se em decréscimos nos níveis de ATT (Figura 3) e na variação dos teores de SS (Figura 4).

Acidez titulável, sólidos solúveis, ratio e pH

$\mathrm{O}$ teste de média mostrou efeito dos dias de armazenamento, sendo $\mathrm{p}<0,001$. O conteúdo de AT aumentou até o $9^{\circ}$ dia de armazenamento, quando ocorreu o pico respiratório, com decréscimos a partir deste instante. Esse resultado está de acordo com os estudos de Pech (2002), em que se menciona que a redução da acidez é decorrência natural da evolução da maturação dos frutos, na qual os ácidos orgânicos são metabolizados na via respiratória e convertidos em moléculas não ácidas. Observou-se também que até o $6^{\circ}$ dia de armazenamento os valores foram superiores para a temperatura ambiente, e, a partir deste momento, o tratamento refrigerado alcançou maiores valores.

Para os teores de SS não houve interação dos dias de armazenamento $(\mathrm{p}=0,31)$, dos tratamentos $(p=0,88)$, ou interação tratamento e dias de armazenamento $(\mathrm{p}=0,77)$. Também como consequência do metabolismo respiratório climatérico, os teores de SS do abacate incrementaram ao longo do período experimental, atingindo valores máximos entre o $3^{\circ}$ e o $6^{\circ}$ dias nos frutos sob temperatura ambiente e no $9^{\circ}$ dia naqueles sob refrigeração. A partir deste instante, prestou-se como substrato energético para a transformação e sobrevivência pós-colheita. Observou-se também um decréscimo mais acentuado, já a partir do $6^{\circ}$ dia, para frutos em condição ambiente (Figura 4).

$\mathrm{O}$ ratio é a relação SS/AT, sendo um parâmetro indicativo de amadurecimento e qualidade do fruto (CHITARRA; CHITARRA, 2005). Observouse para este parâmetro relação inversa com a acidez titulável (Figura 5), em que $\mathrm{r}=-839$ e $\mathrm{p}=0,0$. O teste de média mostrou efeito significativo apenas para os dias de armazenamento $(\mathrm{p}=0,03)$.

$\mathrm{O} \mathrm{pH}$ dos frutos de abacate armazenados sob condição ambiente e refrigeração aumentou durante 0 período de armazenamento, sendo $\mathrm{p}=0,04$ (Figura 6). Este resultado pode ter sido consequência da diminuição de acidez titulável que ocorre normalmente em todos os frutos durante o amadurecimento, conforme descrito também por Chitarra e Chitarra (2005). Nos trabalhos realizados por Daiuto et al. (2010b), em abacate 'Hass', e Oliveira et al. (2000), em abacate 'Fuerte', os autores observaram estabilidade nos valores de $\mathrm{pH}$ durante o armazenamento.

\section{Firmeza}

Para este parâmetro, houve interação entre tratamento e dias de armazenamento, sendo $p=0,03$. A melhor preservação da firmeza dos abacates 'Fuerte' foi observada quando os frutos foram refrigerados, enquanto para os frutos armazenados em temperatura ambiente, o decréscimo foi gradual e constante; para a temperatura de refrigeração, observou-se aumento nos valores de firmeza e queda apenas após o pico respiratório ( $9^{\circ} \mathrm{dia}$ ) (Figura 7). Dessa forma, pode-se reafirmar que a perda de firmeza é normal durante a pós-colheita (BÁEZ-SAÑUDO et al., 2001). Contudo, também se deve levar em consideração que a firmeza é um importante atributo na qualidade dos frutos, já que afeta a resistência ao transporte, ao ataque de microrganismos e à própria característica sensorial dos frutos.

\section{Atividade da enzima polifenoloxidase}

Constatou-se o decréscimo na atividade da PPO durante o período de armazenamento e valores superiores para os frutos mantidos em temperatura ambiente até o $9^{\circ}$ dia (Figura 8). Houve interação dos tratamentos e dias de armazenamento, sendo $\mathrm{p}=0,03$.

A enzima é encontrada praticamente em todos os tecidos vegetais (CÔRREA et al., 2007), e sua atividade depende da espécie, das condições de cultivo e do estádio de maturação (MARTINS et al., 2004). Dessa maneira, verificou-se que a atividade da PPO decresceu ao longo dos 15 dias de armazenamento, independentemente da temperatura. Contudo, o comportamento da enzima PPO é variável e dependente da matriz do fruto. Por exemplo, em maçãs, verificou-se aumento na atividade ao longo do amadurecimento (COSETENG; LEE, 1987), enquanto para pêssegos, assim como observado nos frutos de abacate, o comportamento foi contrário (BASSI; SELLII, 1990).

\section{Coloração da polpa}

Os valores de luminosidade para a polpa dos frutos armazenados em temperatura ambiente variaram de 88,5 a 80,8 do início ao final do experimento. Já para os frutos refrigerados, esse valor ficou entre 84,5 a 88,8 (Figura 9). Para as duas condições de 
armazenamento, observaram-se altos valores de luminosidade, não inferior a $80 \%$. Esse resultado era esperado, já que a análise foi feita logo após o corte do fruto. $\mathrm{O}$ teste de média mostrou que não houve interação dos dias de armazenamento $\mathrm{p}=0,08)$, dos tratamentos $(p=0,12)$ ou do tratamento e dias de armazenamento $(\mathrm{p}=0,06)$.

Para os valores de cor a* e b*, houve interação dos dias de armazenamento, sendo $\mathrm{p}=0,006$.

Os valores de $\mathrm{a}^{*}$ negativos representam a predominância do componente de cor verde na polpa dos frutos. Observou-se redução da cor verde ao longo do período de armazenamento, que foi mais intenso para a polpa dos frutos mantidos sob temperatura ambiente (Figura 10).

Já para a intensidade azul/amarelo, os resultados foram positivos e indicam a presença do componente amarelo na polpa dos frutos. Para este parâmetro de cor, observou-se aumento dos valores até o $9^{\circ}$ dia de armazenamento para os frutos refrigerados e diminuição nos $3^{\circ}$ e $6^{\circ}$ dias para aqueles mantidos em temperatura ambiente. A partir deste momento os valores começaram a diminuir, consequência do início da senescência dos frutos (Figura 11).

Portanto, para as duas condições de armazenamento, ocorreu aumento do componente de cor amarela e diminuição do componente de cor verde, indicando um amarelecimento na polpa.

O escurecimento da polpa de frutos está relacionado à degradação de compostos fenólicos pela PPO que afetam a aparência do produto (SALVETI, 1997); no entanto, nesta pesquisa, não houve correlação significativa entre os parâmetros de cor avaliados e a atividade da PPO. Para a luminosidade e PPO: $\mathrm{p}=$ 0,131 e $r=0,149$; para cor $a^{*}: p=0,957$ e $r=-0,005$ e para cor $b^{*}: p=0,005$ e $r=0,274$.

Golan, Kahn e Sadovski (1977) avaliaram frutos de abacate 'Fuerte' e 'Lerman' e evidenciaram quando a polpa era exposta ao ar, que a razão de escurecimento era maior para os frutos da primeira cultivar. Os autores encontraram uma correlação positiva entre a atividade da PFO com o escurecimento, mas não com conteúdo de o-di-hidroxifenóis (ODHP).

Conteúdo de compostos fenólicos totais e atividade antioxidante

O conteúdo de compostos fenólicos totais aumentou até o $9^{\circ}$ dia de armazenamento para frutos mantidos nas duas condições de armazenamento, descrescendo a partir deste momento em consequência do início do processo de senescência (Figura 12). Até o $5^{\circ}$ dia, os valores foram superiores para frutos refrigerados, e a partir deste ponto esta tendência foi observada para aqueles mantidos em condições de temperatura ambiente. Pelo fato de os frutos estarem mantidos à temperatura ambiente, este aumento da concentração de compostos fenólicos totais poderia estar associado à perda de massa das frutas, concentrando estas substâncias, constatação já feita por Antunes (2006). O mesmo raciocínio pode explicar os valores de atividade antioxidante superiores para a temperatura ambiente

Observaram-se decréscimos após o pico respiratório nos conteúdos de compostos fenólicos totais, cujos valores médios foram de 56,0 e 47,5 $\mu \mathrm{g}$ de GAE. $100 \mathrm{~g}^{-1}$ para os tratamentos ambiente e refrigerado, respectivamente. Os frutos que caracterizaram os menores conteúdos de fenólicos totais foram aqueles submetidos à temperatura de refrigeração. Esse decréscimo pode ser atribuído à série de alterações químicas e enzimáticas de determinados fenóis durante o processo acelerado de amadurecimento desses frutos. Podem-se incluir a essas alterações as hidrólises de glicosídeos por glicosidases, a oxidação de fenóis por fenoloxidases e a polimerização de fenóis livres (ROBARDS et al., 1999). No entanto, não foi encontrada correlação significativa entre a atividade da PFO e o conteúdo de compostos fenólico totais, sendo $\mathrm{p}=0,052$ e $\mathrm{r}=$ $-0,190$. O teste de média mostrou interação para os dias de armazenamento, sendo $\mathrm{p}=0,04$.

Silva et al. (2004) mencionam que a presença desses compostos nos alimentos pode proporcionar efeitos benéficos à saúde humana. Esses dados são concordantes com pesquisas em que é demonstrado que as propriedades de vários compostos fenólicos presentes em frutos atuam com eficácia nas infecções causadas por Helicobacter pylori e na indução da apoptose (YEH; YEN, 2005).

Para AA, houve efeito de dia $(\mathrm{p}<0,001)$. Os valores de atividade antioxidante variaram de 17,6 a $68,7 \%$ (Figura 13). Observaram-se valores crescentes ao longo do armazenamento, mesmo após o pico respiratório. Até o $9^{\circ} \mathrm{dia}$, os valores foram superiores para frutos em temperatura ambiente. Arancibia-Avila et al.(2008) observaram que os polifenóis totais, flavonoides e antocianinas foram significativamente $(\mathrm{p}<0,05)$ superiores nos frutos amadurecidos, comparados aos verdes ou em senescência de durian (Durio zibethinus Murr., cv. Mon Thong).

Apesar de o conteúdo de componentes fenólicos totais não necessariamente estar envolvido na quantificação da atividade antioxidante (JACOBOVELASQUÉZ; CISNEROS-ZEVALLOS, 2009), os resultados dessa análise na polpa dos frutos de abacate 'Fuerte', avaliados por DPPH, apresentaram mesma tendência apenas até o pico respiratório. Para atividade antioxidante e compostos fenólicos, 
a análise de correlação mostrou-se não significativa, em que $p=0,992$ e $r=0,001$, sendo um dos motivos o aumento da atividade antioxidante mesmo após o pico respiratório dos frutos. Já Arancibia-Avila et al. (2008) encontraram correlação de 0,98 entre o conteúdo de compostos fenólicos e a capacidade antioxidante. Os autores concluíram que o alto teor de polifenóis foi principal responsável pela capacidade antioxidante. Wang, Terrell e Bostic (2010) encontraram correlação significativa para o conteúdo de fenólicos totais e a AA $(>=\mathrm{a} 0,79)$. Estes dois parâmetros avaliados pelos autores não se correlacionaram com o conteúdo de clorofila e carotenoides $(\mathrm{r}<0,1)$. Para os autores, a alta correlação entre as procianidinas, o conteúdo de polifenóis e a atividade antioxidante sugere que este composto é o polifenol principal que contribui para a atividade antioxidante em abacate.

Vale ressaltar que a presença de outros compostos presentes no fruto, como a vitamina $\mathrm{E}$, deve ser avaliada, a fim de verificar sua contribuição para a capacidade antioxidante no abacate.

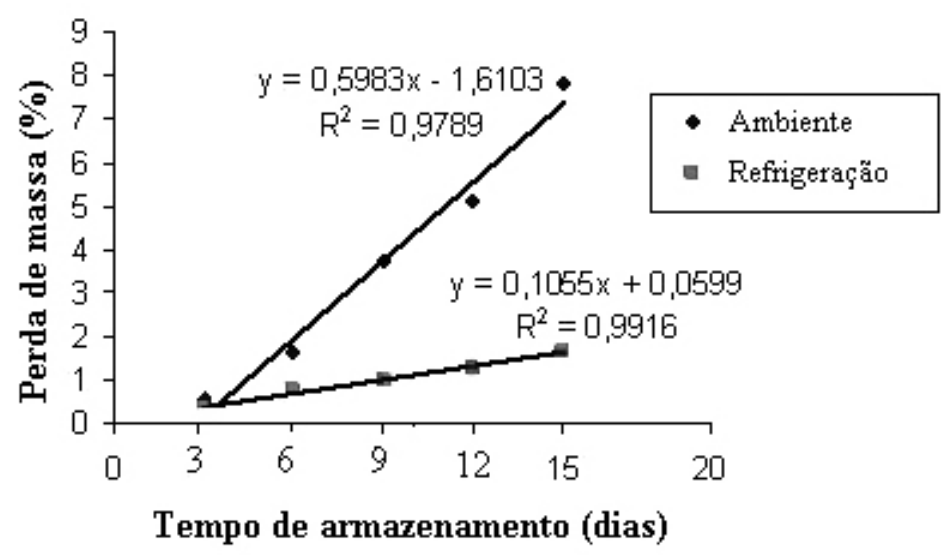

FIGURA 1 - Percentual da perda de massa (\%) em abacate 'Fuerte' armazenado em temperatura ambiente e refrigeração.

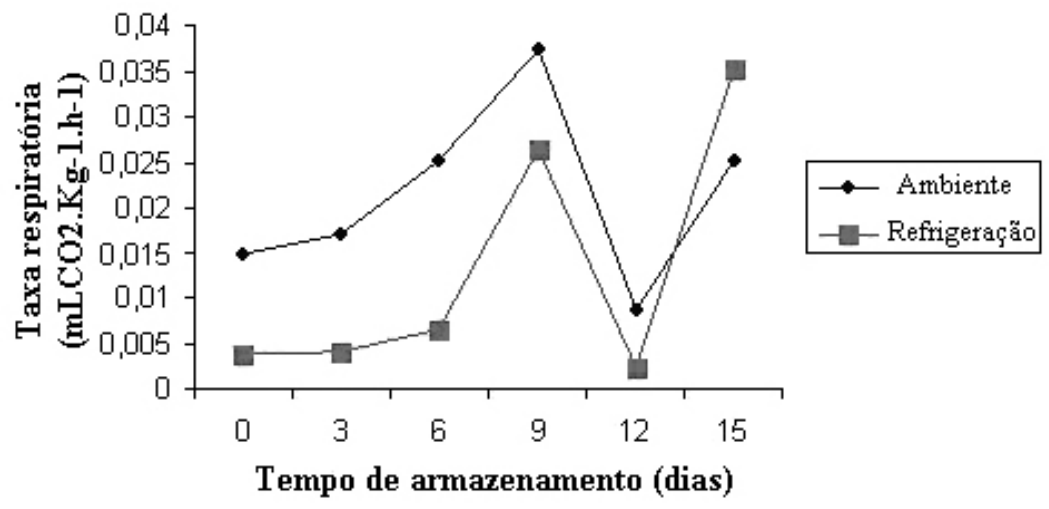

FIGURA 2 - Atividade respiratória $\left(\mathrm{mL}\right.$ de $\left.\mathrm{CO}_{2} \cdot \mathrm{Kg}^{-1} \cdot \mathrm{h}^{-1}\right)$ em abacate 'Fuerte' armazenado em temperatura ambiente e refrigeração. 


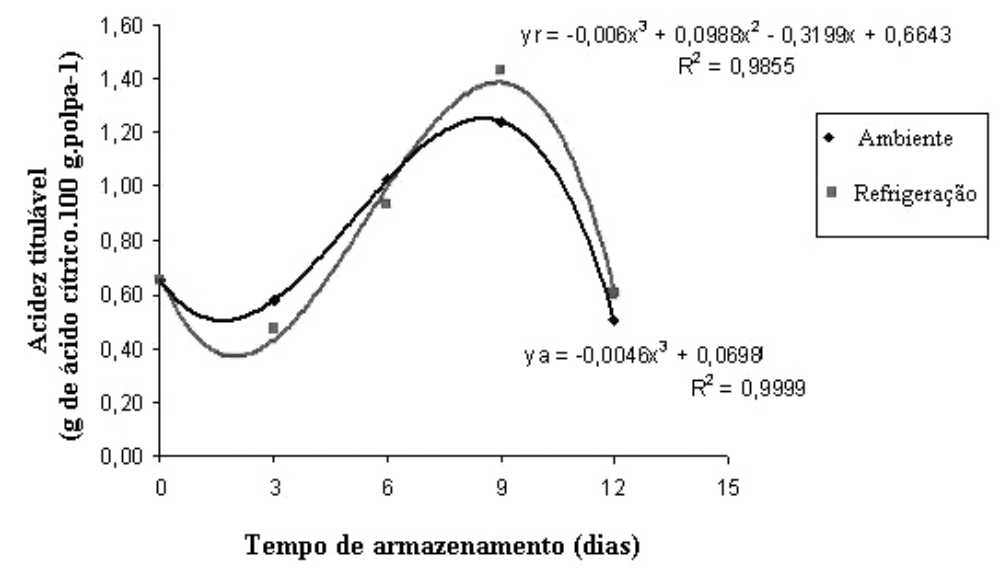

FIGURA 3 - Conteúdo de acidez titulável ( $\mathrm{g}$ de ácido cítrico.100g polpa ${ }^{-1}$ ) em abacate 'Fuerte’ armazenado em temperatura ambiente e refrigeração.

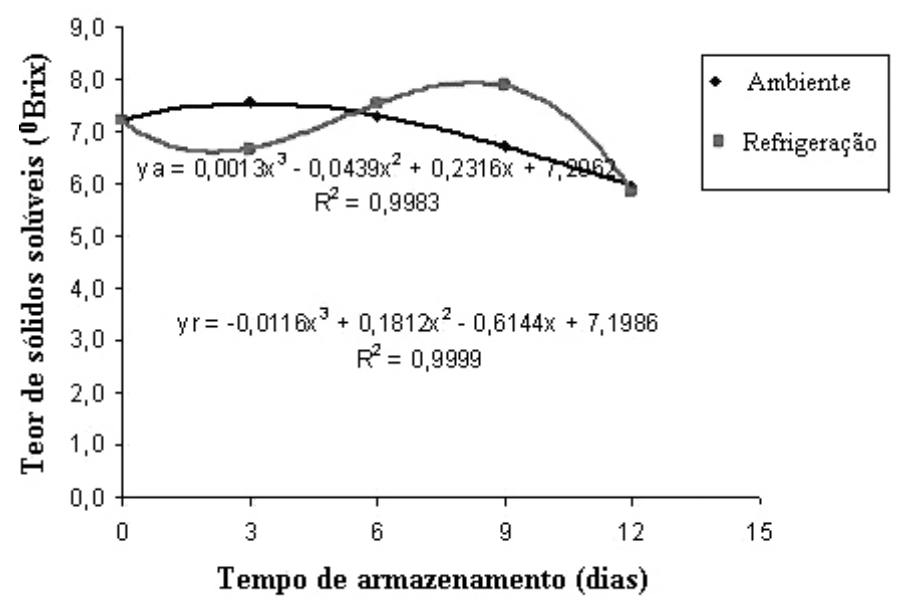

FIGURA 4 - Sólidos solúveis ( ${ }^{\circ}$ Brix) em abacate ‘Fuerte’ armazenado em temperatura ambiente e refrigeração.

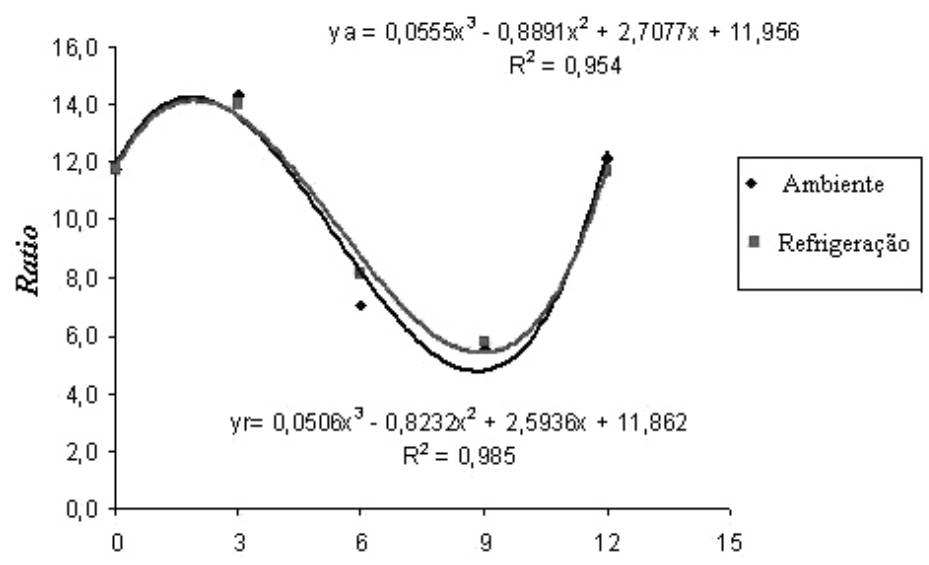

Tempo de armazenamento (dias)

FIGURA 5 - Ratio em abacate 'Fuerte' armazenado em temperatura ambiente e refrigeração. 


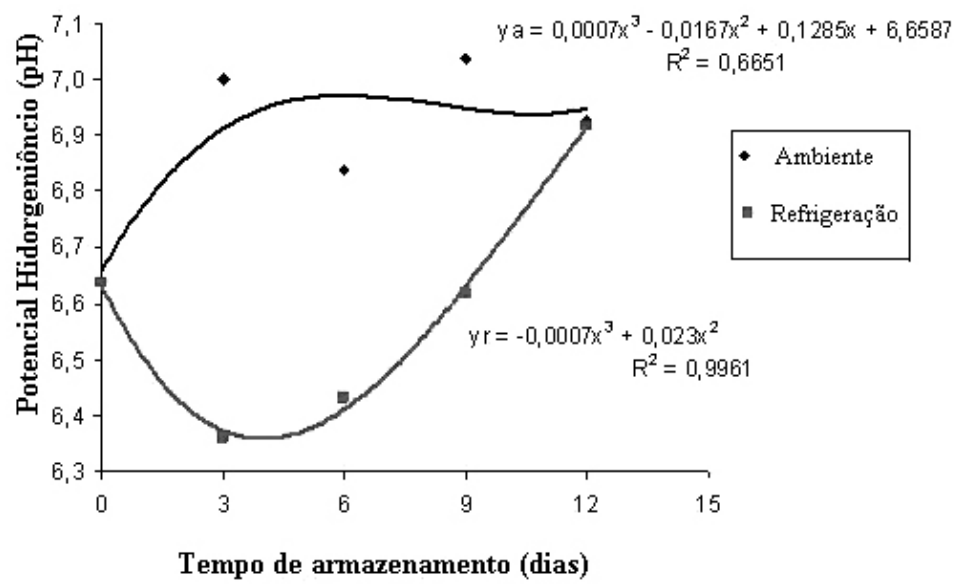

FIGURA 6 - Potencial hidrogeniônico $(\mathrm{pH})$ em abacate 'Fuerte' armazenado em temperatura ambiente e refrigeração.

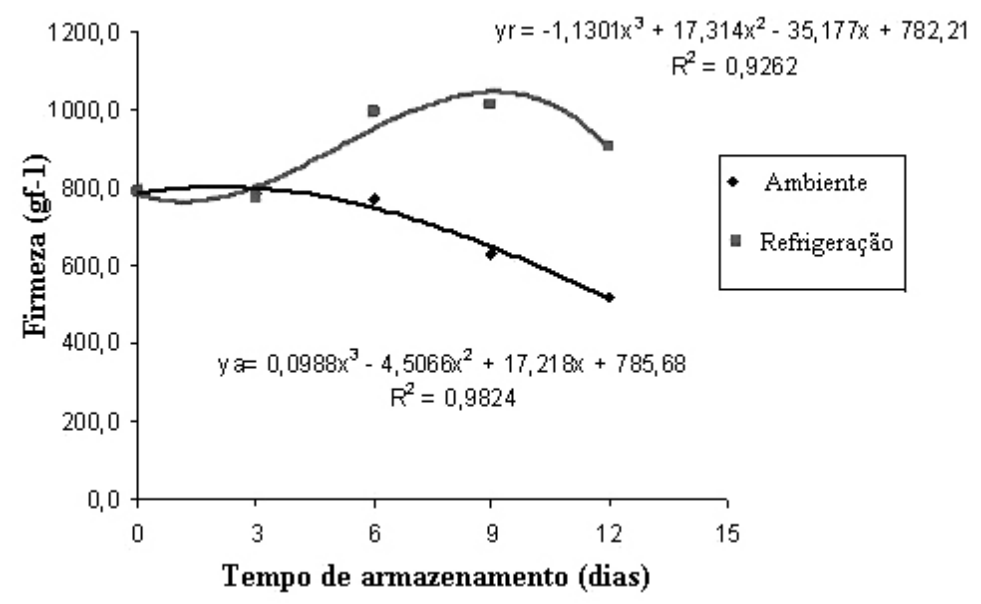

FIGURA 7 - Firmeza (g.f $\left.{ }^{-1}\right)$ em abacate 'Fuerte' armazenado em temperatura ambiente e refrigeração.

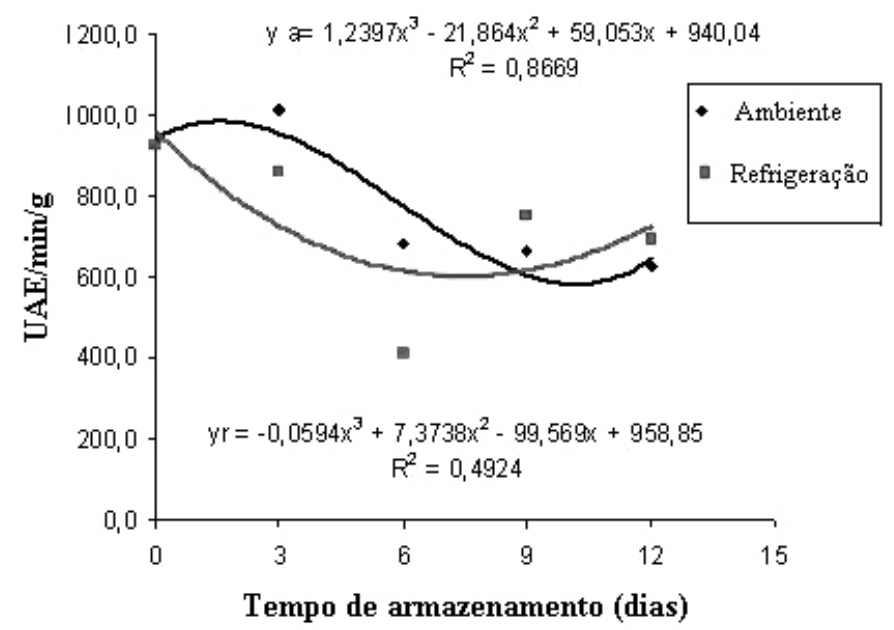

FIGURA 8 - Atividade da polifenoloxidase (UAE/min/g) em abacate 'Fuerte' armazenado em temperatura ambiente e refrigeração. 


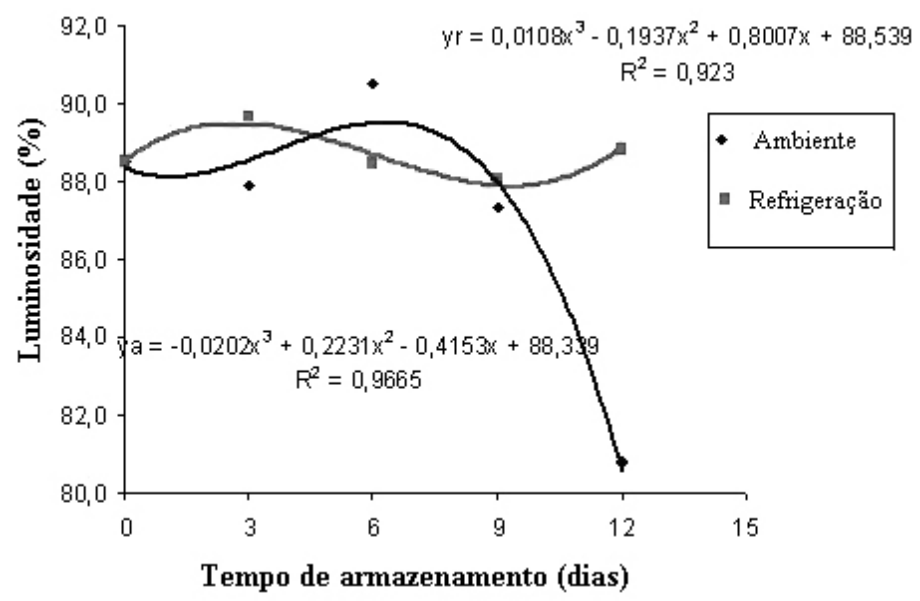

FIGURA 9 - Luminosidade em abacate 'Fuerte' armazenado em temperatura ambiente e refrigeração.

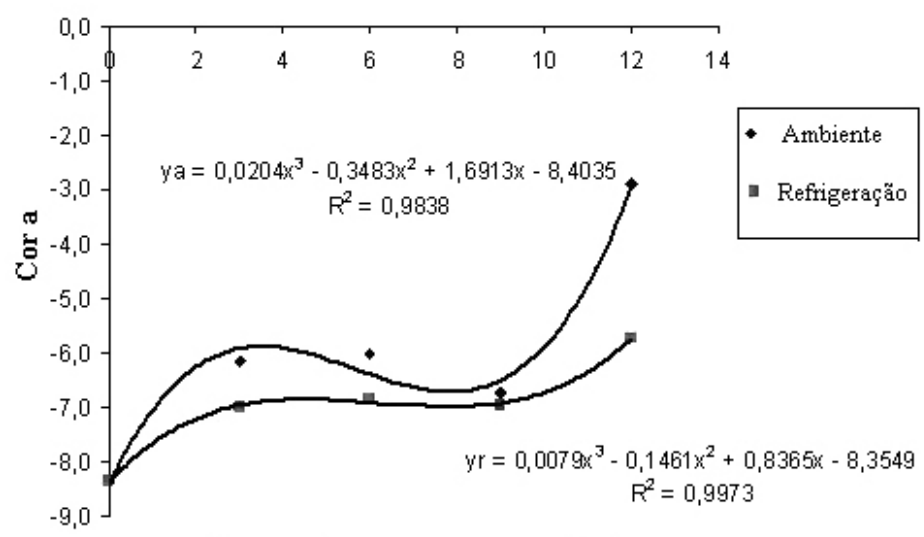

Tempo de armazenamento (dias)

FIGURA 10 - Cor a* em abacate 'Fuerte' armazenado em temperatura ambiente e refrigeração.

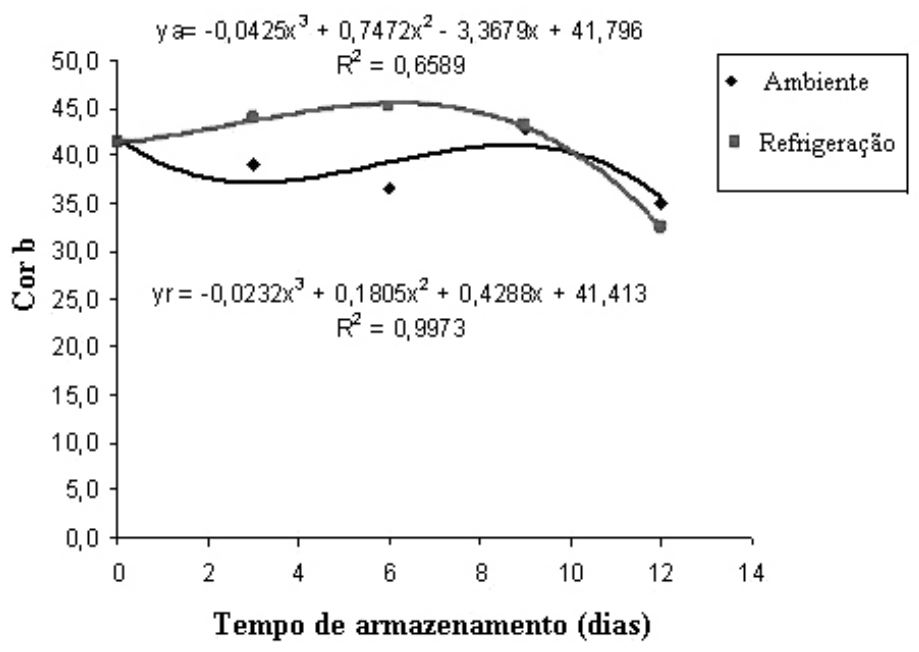

FIGURA 11 - Cor b* em abacate 'Fuerte' armazenado em temperatura ambiente e refrigeração. 


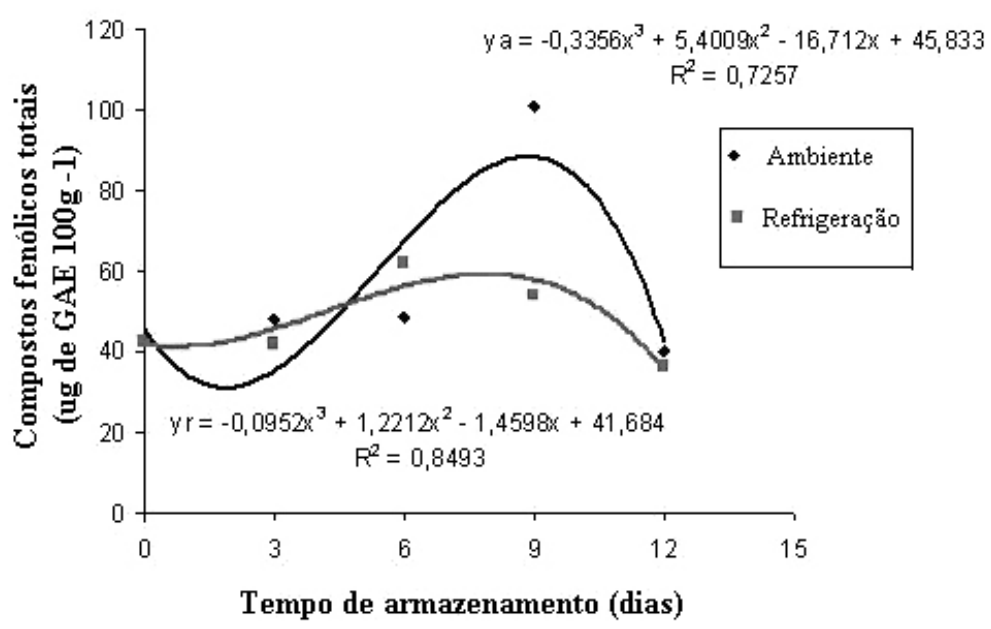

FIGURA 12 - Compostos fenólicos totais ( $\mu \mathrm{g}$ de GAE. $\left.100 \mathrm{~g}^{-1}\right)$ em abacate 'Fuerte' armazenado em temperatura ambiente e refrigeração.

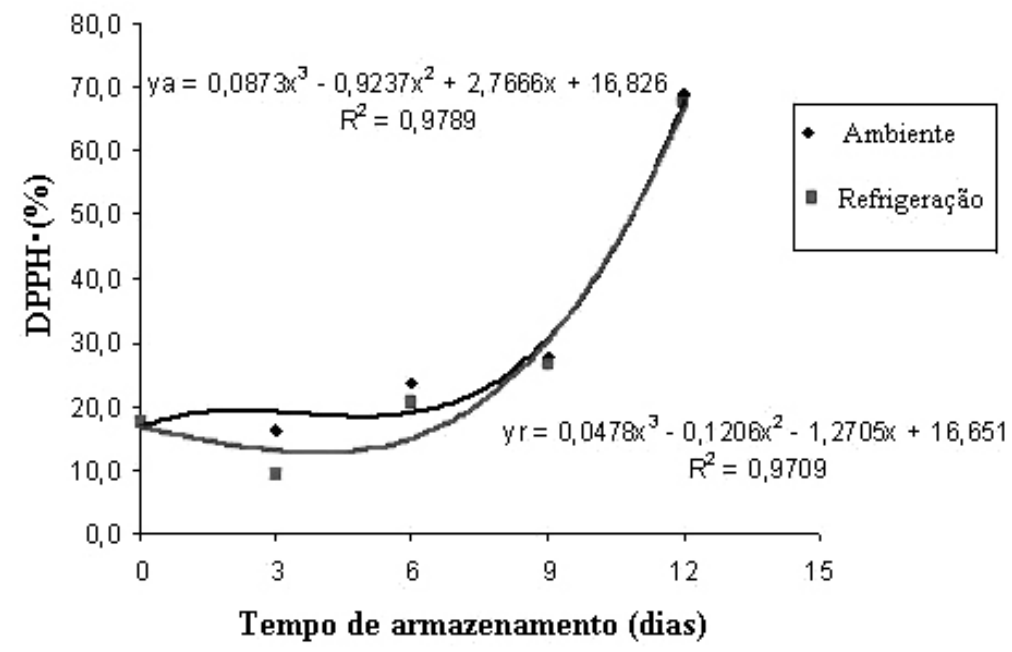

FIGURA 13 - Atividade antioxidante DPPH• em abacate 'Fuerte' armazenado em temperatura ambiente e refrigeração.

\section{CONCLUSÃO}

De acordo com os resultados apresentados, o armazenamento dos frutos 'Fuerte' sob refrigeração é eficiente na manutenção da qualidade pós-colheita, principalmente em relação à perda de massa e firmeza. A capacidade antioxidante aumentou ao longo do período de armazenamento, mesmo após o pico respiratório, e este parâmetro avaliado não apresentou correlação com o conteúdo de compostos fenólicos.

\section{REFERÊNCIAS}

ALI, S. S.; KASOJU, N.; LUTHRA, A.; SINGH, A.; SHARANABASAVA, H.; SAHU, A.; BORA, U. Indian medicinal herbs as sources of antioxidants. Food Research International, Toronto, v. 41, p. 1-15, 2008. 
ANTUNES, L.E.C.; GONÇALVEZ, E.D.; TREVISAN, R. Alterações de compostos fenólicos e pectina em pós-colheita de frutos de amora-preta. Revista Brasileira de Agrociências, Pelotas, v.12, n.1., p.57-61.2006

ARANCIBIA-AVILA, P.; TOLEDO, F.; PARK, Y.S.; JUNG S.T.; KANG, S.G.; HEIO, B.G.; LEE, S.H.; SAJEWICZ, M.; KOWALSKA, T.; GORINSTEIN, $\mathrm{S}$. Antioxidant properties of durian fruit as influenced by ripening. Food Science and Technology, London v.41, p.2118-2125, 2008.

BÁEZ-SAÑUDO, R.; BRINGAS, T. E.; GONZÁLES, A. G.; OJEDA, C. J.; MENDOZA, W. A.; RAMOS, C. G. Evaluación de películas comestibles sobre la vida postcosecha del mango. Proceedings of the International Society Tropical Horticultural, Miami, v.41, p.172-178, 2001.

BASSI, D.; SELLI, R. Evaluation of fruit quality in peach and apricot. Advances in Horticultural Science, Firenze, v.4, p.107-112, 1990.

BLEINROTH, E.W.; ZUCHINI, A.G.; POMPEO, R.M. Determinação das características físicas e mecânicas de variedade de abacate e sua conservação pelo frio. Coletânea ITAL, Campinas, v.7. n.1, p.29-81, 1976.

CANO, M. P.; ANCOS, B. de, MANTALLANA, M. C.; CÁMARA, M.; REGLERO, G.; TABEA, J. Differences among Spanish and Latin-American banana cultivars: morphological, chemical and sensory characteristics. Food Chemistry, Barking, n.59, p.411-419, 1997.

CASTRO, J. V.; BLEINROTH, E. W. Conservação do abacate em atmosfera controlada e à temperatura ambiente. Boletim do Instituto de Tecnologia de Alimentos, Campinas, v. 19, n. 2, p.165-182, 1982.

CHITARRA, M. I. F.;CHITARRA, A. B. Pós-colheita de frutos e hortaliças: fisiologia e manuseio. Lavras: Ed UFLA, 2005. 785 p.

CORRÊA, M. O. G.; PINTO, D. D.; ONO, E. $O$. Análise da atividade respiratória em frutos de jabuticabeira. Revista Brasileira de Biociências, Porto Alegre, v.5, n.2., p.831-833, 2007.
COSETENG, M. Y.; LEE, C. Y. Changes in apple polyphenoloxidase and polyphenol concentrations in relation to degree of browning. Journal of Food Science, New York, v.52, n.4, p.985-989, 1987.

DAIUTO, E.R.; TREMOCOLDI.; VIEITES. M.A.; Conservação pós-colheita de abacate 'hass' irradiado. Revista Iberoamericana de Tecnología Postcosecha, México, v.10, n.2, p.94-100, 2010a.

DAIUTO, E.R.; VIEITES.R.L.; TREMOCOLDI. M.A.; RUSSO, V.C. Taxa respiratória de abacate 'hass' submetido a diferentes tratamentos físicos. Revista Iberoamericana de Tecnología Postcosecha, México, v.10, n.2, p.101-109, 2010 b.

DING, H., CHIN, Y. W., KINGHORN, A. D.; D'AMBROSIO, S. M. Chemopreventive characteristics of avocado fruit. Seminars in Cancer Biology, Stockholm v.17, n.5, p.386-394, 2007.

DONADON, J.R. Distúrbio fisiológico provocado pelo frio e prevenção com tratamentos térmicos em abacates. 2009. 204 f. Tese (Doutorado em Produção Vegetal) - Faculdade de Ciências Agrárias e Veterinárias, Universidade Estadual Paulista, Jaboticabal, 2009.

FINGER, F. L.; VIEIRA, G. Controle da perda póscolheita de água em produtos hortícolas. Viçosa: UFV, 2002. 29 p.

GERMANO, R. M. A.; ARTHUR, V.; WIENDL, F. M. Conservação pós-colheita de abacates Persea americana Mill, variedades Fortuna e Quintal, por irradiação. Scientia Agrícola, Piracicaba, v. 53, n.23, p.249-253, 1996.

GOLAN, A.; KAHN, V.; SADOVSKI, A. Y. Relationship between polyphenols and browning in avocado mesocarp. comparison between the fuerte and lerman cultivars . Journal of Agriculture and Food Chemistry, Easton v. 25, n.6, p.1253-1260, 1977.

HARDENBURG, R. E.; WATADA. A. E.; WANG, $C$. Y. The comercial storage of fruits, vegetables, and florist and nursery stocks. Beltsville: USDA, 1986. 130p.

HEIM, K.E.; TAGLIAFERRO, A.R.; BOBOLYA, D.J. Flavonoid antioxidants: chemistry, metabolism and structure-activity relationships. Journal of Nutritional Biochemistry, Stoneham, v.13, p.572$584,2002$. 
HONÓRIO, S. L.; MORETTI, C. L. Fisiologia pós-colheita de frutas e hortaliças. In: CORTEZ, L. A. B.; HONÓRIO, S. L.; MORETTI, C. L. Resfriamento de frutas e hortaliças. Brasília: Embrapa Hortaliças, 2002. p. 60-81.

JACOBO-VELÁZQUEZ, D. A.; CISNEROSZEVALLOS, L. Correlations of antioxidant activity versus phenolic content revisited: A new approach in data analysis for food and medicinal plants. Journal of Food Science, New York,v.74 n.9, p. R107 - R113, 2009.

KLUGE, R.A.; NACHTIGAL, J.C.; FACHINELLO, J.C.; BILHALVA, A.B. Fisiologia e manejo póscolheita de frutas de clima temperado. 2. ed. Campinas: Livraria e Editora Rural, 214p. 2002.

MANOLOPOULOU, H.; PAPADOPOULOU, P. A study of respiratory and physico-chemical changes of four kiwi fruit cultivars during cool-storage. Food Chemistry, Barking, v.63, p.529-534, 1998.

MARTINS, C. R.; CANTILLANO, R. F. F.; FARIAS, R. de M.; ROMBALDI, C. V. Atividade polifenoloxidase em pós-colheita de pêssegos de pomares de produção integrada e convencional. Ciência Rural, Santa Maria, v. 34, n.3, p.749-754, 2004.

MENSOR, L.L.; MENEZES, F.S.; LEITÃO, G.G.; REIS, A.S.; DOS SANTOS, T.C.; COUBE, C.S.; LEITÃO, S.G. Screening of Brazilian Plant Extracts for Antioxidant Activity by the Use of DPPH Free Radical Method, Phytotherapy Research, London, v.15, p.127-130, 2001.

OLIVEIRA, M.A.de; SANTOS, C.H.; HENRIQUE, C.M.; DOMINGOS, J.R.D. Ceras para conservação pós colheita de frutos de abacateiro fuerte, armazenados em temperatura ambiente. Scientia Agricola, Piracicaba.v.57, n.4, p.777-780, 2000.

PECH, J. C. Unravelling the mechanisms of fruit ripening and development of sensory quality thought the manipulation of ethylene biosynthesis in melon. In: NATO ADVANCED RESEARCH WORSHOPON BIOLOGY AND BIOTECHNOLOGYOF THE PLANT HORMONE ETHYLENE, 2002, Murcia. Anais...

ROBARDS, K.; PRENZLER, P. D.; TUCKER, G.; SWATSITANG, P.; GLOVER, W. Phenolic compounds and their role in oxidative processes in fruits. Food Chemistry, Barking, v.66, p. 401-436, 1999.
SALVEIT, M.E. Physical and physiological changes in minimally processed fruits and vegetables. In: TOMÁS-BARBERÁN F.A.; ROBIN R.D. (Ed.). Phytochemestry of fruits and vegetables. Oxford: Oxford University Press, 1997. p.205-220.

SEYMOUR, G. B.; TUCKER, G. A. Avocado. In: SEYMOUR, G. B.; TAYLOR, J. E.; TUCKER, G. A. Biochemistry of fruit ripening. London: Chapman \& Hall, 1993. p. 53-76.

SILVA, B. M.; ANDRADE, P. B.; VALENTAO, P.; FERRERES, F.; SEBRA, R. M.; FERREIRA, M. Quince (Cydonia oblonga Miller) fruit (pulp, peel, and seed) and jam: Antioxidant activity. Journal of Agriculture and Food Chemistry, Easton, n.52, p.4705-4712. 2004

SINGLETON, V. L.; ORTHOFER, R.; LAMUELA, R. M. Analysis of totalphenols and other oxidation substrates and antioxidants by means of Folin-Ciocalteau reagent. Methods of Enzymology,

SOARES, D.G.; ANDREAZZA, A.C.; SALVADOR, M. Avaliação de compostos com atividade antioxidante em células da levedura Saccharomyces cerevisiae. Revista Brasileira de Ciências Farmacêuticas, Araraquara v.41, n.1,p.95-100, 2005.

TANGO, J. S.; CARVALHO, C. R. L.; SOARES, N. B. Caracterização física e química de frutos de abacate visando a seu potencial para extração de óleo. Revista Brasileira de Fruticultura, Jabuticabal, v.6, n.1, p, 17-23, 2004.

TEIXEIRA, C.G.; BLEINROTH, E. W.; CASTRO, J. V.; MARTIN, Z. J.; TANGO, J. S.; TURATTI, J. M.; LEITE, R. S. S. F.; CASTRO, A. E. B. Abacate: cultura, matéria-prima, processamento e aspectos econômicos. Campinas: ITAL, 1991. 250p.

TOMÁS-BARBERÁN, F.A.; ESPÍN, J.C. Phenolic compounds and related enzymes as determinants ofquality in fruits and vegetables. Journal of Science and Food Agriculture, Chicago, n. 81, p. 853-879, mar. 2001.

TRESSLER, D.K.; JOSLYN, M.A. Fruits and vegetables juice processing technogy. Westport: Conn. Avi. 1961, 1028p. 
USDA - U.S. Department of Agriculture, Agricultural Research Service. USDA national nutrient database for standard reference: release 20 . 2007. Disponível em: <http://www.ars.usda.gov/ $\mathrm{ba} / \mathrm{bhnrc/ndl}>$.

WANG, W.; TERRELL, R.; BOSTIC, L.G. Antioxidant capacities, procyanidins and pigments in avocados of different strains and cultivars. Food Chemistry, Barking, v.122, p. 1193-1198, 2010.

YEH, C. T.; YEN G. C. Induction of apoptosis by the anthocyanidins through regulation of $\mathrm{Bcl}-2$ gene and activation of c-jun n-terminal kinase cascade in hepatoma cells. Journal of Agriculture and Food Chemistry, Easton, v.53, p.1740-1749, 2005.
ZAUBERMAN, M. S.; SCHIFFMAN-NADEL, M.;YANKO, U.Susceptibility to chilling injury of three avocado cultivars stages of ripening. HortScience, Alexandria, v. 8, n. 4, p. 511-513, 1973.

ZENEBON, O.; PASCUET, N.S., TIGLEA, P. (Coord.). Métodos físico-químicos para análise de alimentos. São Paulo: Instituto Adolfo Lutz, 2008. p. 1020. versão eletrônica. 